\title{
Dynamic Behaviors of a Leslie-Gower Ecoepidemiological Model
}

\author{
Aihua Kang, ${ }^{1,2}$ Yakui Xue, ${ }^{1,3}$ and Jianping Fu ${ }^{1}$ \\ ${ }^{1}$ College of Mechatronic Engineering, North University of China, Taiyuan, Shanxi 030051, China \\ ${ }^{2}$ Shuozhou Advanced Normal College, Shuozhou, Shanxi 036000, China \\ ${ }^{3}$ Department of Mathematics, North University of China, Taiyuan, Shanxi 030051, China
}

Correspondence should be addressed to Aihua Kang; kangaihua1982@163.com

Received 11 August 2015; Accepted 2 November 2015

Academic Editor: Yi Wang

Copyright (C) 2015 Aihua Kang et al. This is an open access article distributed under the Creative Commons Attribution License, which permits unrestricted use, distribution, and reproduction in any medium, provided the original work is properly cited.

\begin{abstract}
A Leslie-Gower ecoepidemic model with disease in the predators is constructed and analyzed. The total population is subdivided into three subclasses, namely, susceptible predator, infected predator, and prey population. The positivity, boundness of solutions, and the existence of the equilibria are studied, and the sufficient conditions of local asymptotic stability of the equilibria are obtained by the Routh-Hurwitz criterion. We analyze the global stability of the interior equilibria by using Lyapunov functions. It is observed that a Hopf bifurcation may occur around the interior equilibrium. At last, numeric simulations are performed in support of the feasibility of the main result.
\end{abstract}

\section{Introduction}

Since the pioneering work of Anderson and May [1], many researchers have paid great attention to the modeling and analysis of ecoepidemiological systems recently. Venturino [2], Haque et al. [3], Xiao and Chen [4, 5], Tewa et al. [6], Rahman and Chakravarty [7], and so forth discussed the dynamics of prey-predator system with disease in prey population. Haque et al. [8] analyzed the dynamical behavior of predator-prey system with disease in predator population. Hsieh and Hsiao [9] proposed and discussed the dynamics of a predator-prey model with disease in both prey and predator populations. The boundness and stability of the equilibria are studied. There are mainly two types functional response: Holling-type functional response and Leslie-Gower functional response. Most scholars discussed the Hopf bifurcation and the Bogdanov-Takens bifurcation near the boundary equilibrium.

The Leslie-Gower functional response is first proposed by Leslie [10], which introduced the following predator-prey model where the "carrying capacity" of the predator's environment is proportional to the number of prey populations.
The first and second Leslie-Gower predator-prey models are as follows:

$$
\begin{aligned}
\frac{d H}{d t} & =\left(r_{1}-a_{1} P\right) H, \\
\frac{d P}{d t} & =\left(r_{2}-a_{2} \frac{P}{H}\right) P, \\
\frac{d H}{d t} & =\left(r_{1}-a_{1} P-b_{1} H\right) H, \\
\frac{d P}{d t} & =\left(r_{2}-a_{2} \frac{P}{H}\right) P,
\end{aligned}
$$

where $H$ and $P$ are the density of prey species and the predator species at time $t$, respectively. Because of the complex mathematical expressions involved in the analysis, Korobeinikov [11] introduced a Lyapunov function for both models (1) and (2) to prove their global stabilities. After the work of Korobeinikov, many scholars have done works on Leslie-type predator prey ecosystem. The modified Leslie-Gower and Holling-type II predator-prey model is generalized in the context of ecoepidemiology, with disease spreading only among the prey species [12]. Hopf bifurcation 
is studied for a modified Leslie-Gower predator-prey system with harvesting [13]. Aziz-Alaoui [14] studied dynamic behaviors of three Leslie-Gower-type species food chain systems. Chen et al. [15] incorporated a prey refuge to system (1) and showed that the refuge has no influence on the persistent property of the system. A predator-prey LeslieGower model with disease in prey has been developed, where it is observed that a Hopf bifurcation may occur around the interior equilibrium taking refuge parameter as bifurcation parameter [16]. Some similar kinds of models have appeared in the recent literature; the main new distinctive feature is the inclusion of an infectious disease in prey population. But the disease also can spread in predator because of food, parasite, mating, and so on.

In the present research, we formulate a predator-prey Leslie-Gower model with disease in predator. The total population have been divided into three classes, namely, susceptible predator, infected predator, and prey population. The construction and model assumptions are discussed in Section 2. In Section 3, positivity and boundedness of the solutions of the model are discussed. Section 4 deals with their existence and stability analysis of the equilibrium points. In Section 5, a detailed study of the Hopf bifurcation around the interior equilibrium is carried out. Numerical illustrations are performed finally in order to validate the applicability of the model under consideration.

\section{The Mathematical Model}

We construct the following model:

$$
\begin{aligned}
& \frac{d X}{d t}=r_{1} X\left(1-\frac{X}{k}\right)-a X Y_{1}, \\
& \frac{d Y_{1}}{d t}=r_{2} Y_{1}\left[1-\frac{h\left(Y_{1}+Y_{2}\right)}{X}\right]-\beta Y_{1} Y_{2}-d_{1} Y_{1}, \\
& \frac{d Y_{2}}{d t}=\beta Y_{1} Y_{2}-d_{2} Y_{2}
\end{aligned}
$$

with initial conditions

$$
\begin{gathered}
X(0) \geq 0, \\
Y_{1}(0) \geq 0, \\
Y_{2}(0) \geq 0,
\end{gathered}
$$

where $X(t), Y_{1}(t)$, and $Y_{2}(t)$ are the density of prey, susceptible predator, and infected predator populations, respectively, at time $t$. The prey population grows according to a logistic fashion with carrying capacity $k$ and an intrinsic birth rate constant $r_{1}, r_{2}$ is the intrinsic growth rate of susceptible predator populations. $\beta$ is the transmission coefficient from susceptible predator to infected predator. $h$ is the maximum value of the per capita reduction rate of $X$ due to $Y=\left(Y_{1}+Y_{2}\right)$. The second equation of system (3) contains the so-called Leslie-Gower term, namely, $h\left(Y_{1}+Y_{2}\right) / X . d_{1}$ is the natural death rate of susceptible predator. $d_{2}$ is death rate of infected predator including natural death rate and disease related death rate in the absence of predator. The model parameters $r_{1} ; r_{2} ; k ; a ; h ; \beta ; d_{1}$; and $d_{2}$ are all positive constants.

\section{Some Preliminary Results}

Theorem 1. Every solution of system (3) with initial conditions (4) exists in the interval $[0,+\infty)$ and $X(t) \geq 0, Y_{1}(t) \geq 0$, $Y_{2}(t) \geq 0$ for all $t>0$.

Proof. Since the right-hand side of system (3) is completely continuous and locally Lipschitzian on $C$, the solution $\left(X(t), Y_{1}(t), Y_{2}(t)\right)$ of (3) with initial conditions (4) exists and is unique on $[0, \zeta)$, where $0<\zeta \leq+\infty$ [17]. From system (3) with initial conditions (4), we have

$$
\begin{aligned}
& X(t) \geq X(0) \int_{0}^{+\infty} r_{1}\left[X(s)\left(1-\frac{X(s)}{k}\right)\right. \\
& \left.\quad-a X(s) Y_{1}(s)\right] d s \geq 0 \\
& Y_{1}(t) \geq Y_{1}(0) \int_{0}^{+\infty}\left\{r_{2} Y_{1}(s)\left[1-\frac{h\left(Y_{1}(s)+Y_{2}(s)\right)}{X(s)}\right]\right. \\
& \left.\quad-\beta Y_{1}(s) Y_{2}(s)-d_{1} Y_{1}(s)\right\} d s \geq 0, \\
& Y_{2}(t) \geq Y_{2}(0) \int_{0}^{+\infty}\left[\beta Y_{1}(s) Y_{2}(s)-d_{2} Y_{2}(s)\right] d s \geq 0
\end{aligned}
$$

which completes the proof.

Theorem 2. All solutions of system (3) initiating $R^{3}$ are ultimately bounded.

Proof. We consider first $X(t) \leq k, \forall t>0$ :

$$
\frac{d X}{d t}=r_{1} X\left(1-\frac{X}{k}\right)-a X Y_{1} \leq r_{1} X\left(1-\frac{X}{k}\right) \text {. }
$$

We get $X=1 /\left(C e^{-r_{1} t}+1 / k\right)$. If $t \rightarrow \infty, X \rightarrow k$ :

$$
\begin{aligned}
\frac{d Y_{1}}{d t}+\frac{d Y_{2}}{d t} & =r_{2} Y_{1}\left[1-\frac{h\left(Y_{1}+Y_{2}\right)}{X}\right]-d_{1} Y_{1}-d_{2} Y_{2} \\
& \leq r_{2} Y_{1}\left[1-\frac{h\left(Y_{1}+Y_{2}\right)}{X}\right] \\
& \leq r_{2} Y_{1}\left[1-\frac{h\left(Y_{1}+Y_{2}\right)}{k}\right] \\
& \leq r_{2}\left(Y_{1}+Y_{2}\right)-\frac{r_{2} h}{k}\left(Y_{1}+Y_{2}\right)^{2} .
\end{aligned}
$$

So again by solving the above linear differential inequality, we have

$$
0<Y_{1}+Y_{2}<\frac{1}{e^{-r_{2} t}+h / k}
$$

If $t \rightarrow \infty, Y_{1}+Y_{2} \rightarrow h / k$. The proof is completed.

Therefore, the feasible region $\Gamma$ defined by

$\Gamma$

$$
=\left\{\left(X(t), Y_{1}(t), Y_{2}(t)\right) \in R_{+}^{3}: X \leq k, Y_{1}+Y_{2} \leq \frac{h}{k}\right\}
$$


with $X(0) \geq 0, Y_{1}(0) \geq 0$, and $Y_{2}(0) \geq 0$ is positively invariant of model (3).

\section{Stability Analysis}

4.1. Existence of Equilibrium Points. All equilibrium points of system (3) are as follows:

(1) trivial equilibrium: $E_{0}=(0,0,0)$;

(2) axial equilibrium: $E_{1}=(k, 0,0)$;

(3) if $r_{2}>d_{1}$, the planar equilibrium $E_{2}=\left(k r_{2} h r_{1} /\right.$ $\left.\left(r_{1} r_{2} h+a k\left(r_{2}-d_{1}\right)\right), k r_{1}\left(r_{2}-d_{1}\right) /\left(r_{1} r_{2} h+a k\left(r_{2}-d_{1}\right)\right), 0\right)$ exists;

(4) if $\beta r_{1}>a d_{2}$, and $k\left(r_{2}-d_{1}\right)\left(\beta r_{1}-a d_{2}\right)-r_{2} h r_{1} d_{2}>$ 0 , the interior equilibrium $E_{*}=\left(X_{*}, Y_{1 *}, Y_{2 *}\right)$ exists, where $X_{*}=k\left(\beta r_{1}-a d_{2}\right) / \beta r_{1}, Y_{1 *}=d_{2} / \beta$, and $Y_{2 *}=$ $\left(k\left(r_{2}-d_{1}\right)\left(\beta r_{1}-a d_{2}\right)-r_{2} h r_{1} d_{2}\right) /\left(r_{2} h \beta r_{1}+\beta\left(k \beta r_{1}-\right.\right.$ $\left.\left.\operatorname{kad}_{2}\right)\right)$.

4.2. Local Stability. Let $\bar{E}=\left(\bar{X}, \bar{Y}_{1}, \bar{Y}_{2}\right)$ be an equilibrium point of model (3); the Jacobian matrix of system (3) at the equilibrium point is

$$
\left(\begin{array}{ccc}
r_{1}-\frac{2 r_{1}}{k} \bar{X}-a \bar{Y}_{1} & -a \bar{X} & 0 \\
\frac{h r_{2} \bar{Y}_{1}\left(\bar{Y}_{1}+\bar{Y}_{2}\right)^{2}}{\bar{X}} & r_{2}-\frac{2 r_{2} h \bar{Y}_{1}}{\bar{X}} & -\frac{r_{2} h \bar{Y}_{1}}{\bar{X}}-\beta \bar{Y}_{1} \\
0 & \beta \bar{Y}_{2} & \beta \bar{Y}_{1}-d_{2}
\end{array}\right)
$$

Through judging the positive or negative of the eigenvalues which is the characteristic equation, we can know local asymptotic stability of all equilibrium points. Through calculation, we have the following results.

(I) Eigenvalues of the characteristic equation of $E_{1}$ are $\lambda_{1}=-r_{1}, \lambda_{2}=r_{2}-d_{1}$, and $\lambda_{3}=-d_{2}$. It is clear that if $r_{2}<d_{1}, \lambda_{2}<0$, the equilibrium point $E_{1}$ is locally asymptotically stable.

(II) The variational matrix of system (3) at $E_{2}=\left(X_{2}, Y_{12}\right.$, 0 ) is given by

$$
\left(\begin{array}{ccc}
-\frac{r_{1}}{k} X_{2} & -a X_{2} & 0 \\
h r_{2} Y_{12}^{2} & -\frac{r_{2} h Y_{12}}{X_{2}} & -\frac{r_{2} h Y_{12}}{X_{2}}-\beta Y_{12} \\
0 & 0 & \beta Y_{12}-d_{2}
\end{array}\right)
$$

With regard to the equilibrium point $E_{2}$, its characteristic equation is

$$
\mu^{3}+A_{1} \mu^{2}+B_{2} \mu+A_{3}=0,
$$

where $A_{1}=-\left(a_{11}+a_{22}+a_{33}\right), A_{2}=a_{11} a_{12}-a_{12} a_{21}+$ $a_{11} a_{33}+a_{22} a_{33}, A_{3}=-a_{33}\left(a_{11} a_{12}-a_{12} a_{21}\right)$ and $a_{11}=$ $-k r_{2} h r_{1}^{2} /\left(r_{1} r_{2} h+a k\left(r_{2}-d_{1}\right)\right), a_{12}=-a k r_{2} h r_{1} /\left(r_{1} r_{2} h+\right.$ $\left.a k\left(r_{2}-d_{1}\right)\right), a_{21}=\left(r_{2}-d_{1}\right)^{2} / r_{2} h, a_{22}=-\left(r_{2}-d_{1}\right) /$ $\left(r_{1} r_{2} h+a k\left(r_{2}-d_{1}\right)\right)$, and $a_{33}=\beta k r_{1}\left(r_{2}-d_{1}\right) /\left(r_{1} r_{2} h+\right.$ $\left.a k\left(r_{2}-d_{1}\right)\right)-d_{2}$.
If $k\left(r_{2}-d_{1}\right)\left(\beta r_{1}-a d_{2}\right)-r_{2} h r_{1} d_{2}<0, a_{33}<0$. Obviously, $A_{1}>0, A_{3}>0$, and $A_{1} A_{2}-A_{3}>0$.

By the Routh-Hurwitz rule, the equilibrium point $E_{2}$ is locally asymptotically stable in the region $\Gamma$.

(III) The variational matrix of system (3) at $E_{*}=$ $\left(X_{*}, Y_{1 *}, Y_{2 *}\right)$ is given by

$$
\left(\begin{array}{ccc}
-\frac{r_{1}}{k} X_{*} & -a X_{*} & 0 \\
\frac{h r_{2} Y_{1 *}\left(Y_{1 *}+Y_{2 *}\right)}{X_{*}^{2}} & \frac{r_{2} h\left(Y_{2 *}-Y_{1 *}\right)}{X_{*}} & -\frac{r_{2} h Y_{1 *}}{X_{*}}-\beta Y_{1 *} \\
0 & \beta Y_{2 *} & 0
\end{array}\right)
$$

With regard to the equilibrium point $E_{*}$, its characteristic equation is

$$
\mu^{3}+B_{1} \mu^{2}+B_{2} \mu+B_{3}=0,
$$

where $B_{1}=-\left(b_{11}+b_{22}\right), B_{2}=b_{11} b_{22}-b_{12} b_{21}-b_{23} b_{32}$, $B_{3}=b_{11} b_{23} b_{32}$ and $b_{11}=-\left(\beta r_{1}-a d_{2}\right) / \beta, b_{12}=$ $-a k\left(\beta r_{1}-a d_{2}\right) / \beta r_{1}, b_{21}=\beta r_{1}^{2} h r_{2}\left(1+r_{2}-d_{1}\right) / k\left(\beta r_{1}-\right.$ $\left.a d_{2}\right)\left(r_{2} h r_{1}+k\left(\beta r_{1}-a d_{2}\right)\right), b_{22}=r_{2} h r_{1}\left(k r_{2} \beta r_{1}+\right.$ $\left.k d_{1} a d_{2}+k a d_{2}^{2}-k r_{2} a d_{2}-k d_{1} \beta r_{1}-2 r_{1} r_{2} h d_{2}-d_{2} k r_{1} \beta\right) /$ $k\left(\beta r_{1}-a d_{2}\right)\left(r_{2} h r_{1}+k\left(\beta r_{1}-a d_{2}\right)\right), b_{23}=-\left(r_{1} r_{2} h d_{2}+\right.$ $\left.d_{2} k\left(\beta r_{1}-a d_{2}\right)\right) / k\left(\beta r_{1}-a d_{2}\right)$, and $b_{32}=\left(k\left(r_{2}-\right.\right.$ $\left.\left.d_{1}\right)\left(\beta r_{1}-a d_{2}\right)-r_{2} h r_{1} d_{2}\right) /\left(r_{2} h r_{1}+\left(k \beta r_{1}-k a d_{2}\right)\right)$.

If $k\left(r_{2}-d_{1}\right)\left(\beta r_{1}-a d_{2}\right)-r_{2} h r_{1} d_{2}>0, b_{32}>0$, and $B_{3}>0$.

If $Y_{2 *}-Y_{1 *}<0$, in other words $k r_{2} \beta r_{1}+k d_{1} a d_{2}+$ $k a d_{2}^{2}>k r_{2} a d_{2}+k d_{1} \beta r_{1}+2 r_{1} r_{2} h d_{2}+d_{2} k r_{1} \beta, b_{22}<0$, and $B_{1}>0$.

$B_{1} B_{2}-B_{3}=-b_{11}\left(b_{11} b_{22}-b_{12} b_{21}\right)+b_{22}\left(b_{12} b_{21}+b_{23} b_{32}\right)>$ 0 .

By the Routh-Hurwitz rule, the equilibrium point $E_{*}$ is locally asymptotically stable in the region $\Gamma$.

So, we come to the following results.

Theorem 3. If $k\left(r_{2}-d_{1}\right)\left(\beta r_{1}-a d_{2}\right)-r_{2} h r_{1} d_{2}<0$, the planar equilibrium point $E_{2}$ is locally asymptotically stable.

Theorem 4. If $k r_{2} \beta r_{1}+k d_{1} a d_{2}+k a d_{2}^{2}<k r_{2} a d_{2}+k d_{1} \beta r_{1}+$ $2 r_{1} r_{2} h d_{2}+d_{2} k r_{1} \beta$, the interior equilibrium point $E_{*}$ is locally asymptotically stable.

4.3. Globally Asymptotically Stable. Defining the Lyapunov function, we can judge the global asymptotically stability of the interior equilibrium point.

Theorem 5. The equilibrium point $E_{*}$ is locally asymptotically stable, meaning that it is globally asymptotically stable in $\Sigma=$ $\{(S, I, Y): S>0, I>0, Y>0\}$.

Proof. Construct the Lyapunov function

$$
\begin{aligned}
V\left(X, Y_{1}, Y_{2}\right)= & V_{1}\left(X, Y_{1}, Y_{2}\right)+V_{2}\left(X, Y_{1}, Y_{2}\right) \\
& +V_{3}\left(X, Y_{1}, Y_{2}\right)
\end{aligned}
$$


where $V_{1}\left(X, Y_{1}, Y_{2}\right)=X-X_{*}-X_{*} \ln \left(X / X_{*}\right), V_{2}\left(X, Y_{1}, Y_{2}\right)=$ $Y_{1}-Y_{1 *}-Y_{1 *} \ln \left(Y_{1} / Y_{1 *}\right)$, and $V_{3}\left(X, Y_{1}, Y_{2}\right)=Y_{2}-Y_{2 *}-$ $Y_{2 *} \ln \left(Y_{2} / Y_{2 *}\right)$

Since the solutions of the system are bounded and ultimately enter the set $\Gamma$, we restrict the study for this set. The time derivative of $V_{1}$ along the solutions of system (3) is

$$
\begin{aligned}
\frac{d V_{1}}{d t} & =\frac{X-X_{*}}{X}\left[r_{1}-\frac{X}{k}-a Y_{1}\right] X \\
& =-\frac{1}{k}\left(X-X_{*}\right)^{2}-a\left(X-X_{*}\right)\left(Y_{1}-Y_{1 *}\right) .
\end{aligned}
$$

Similarly,

$$
\begin{aligned}
\frac{d V_{2}}{d t}= & \frac{Y_{1}-Y_{1 *}}{Y_{1}}\left[r_{2}-\frac{r_{2} h\left(Y_{1}+Y_{2}\right)}{X}-\beta Y_{2}-d_{1}\right] Y_{1} \\
= & -\left(\beta+\frac{r_{2} h}{X}\right)\left(Y_{1}-Y_{1 *}\right)\left(Y_{2}-Y_{2 *}\right) \\
& -\frac{r_{2} h}{X}\left(Y_{1}-Y_{1 *}\right)^{2} \\
& +\frac{r_{2} h\left(Y_{1 *}+Y_{2 *}\right)}{X X_{*}}\left(X-X_{*}\right)\left(Y_{1}-Y_{1 *}\right), \\
\frac{d V_{3}}{d t}= & \beta\left(Y_{1}-Y_{1 *}\right)\left(Y_{2}-Y_{2 *}\right) .
\end{aligned}
$$

The above equation can be written as

$$
\begin{aligned}
\frac{d V}{d t} & =-\left(X-X_{*}, Y_{1}-Y_{1 *}, Y_{2}-Y_{2 *}\right) \\
& \left(\begin{array}{ccc}
\frac{1}{k} & -g\left(X, Y_{1}, Y_{2}\right) & 0 \\
g\left(X, Y_{1}, Y_{2}\right) & \frac{r_{2} h}{X} & \frac{r_{2} h}{2 X} \\
0 & \frac{r_{2} h}{2 X} & 0
\end{array}\right)\left(\begin{array}{c}
X-X_{*} \\
Y_{1}-Y_{1 *} \\
Y_{2}-Y_{2 *}
\end{array}\right),
\end{aligned}
$$

where $g\left(X, Y_{1}, Y_{2}\right)=(1 / 2)\left[a+r_{2} h\left(Y_{1 *}+Y_{2 *}\right) / X X_{*}\right]$.

This matrix is positive definite if all upper-left submatrices are positive.

Through calculating all upper-left submatrices:

$$
\begin{aligned}
M_{1} & =\left|\begin{array}{cc}
\frac{1}{k} & -g\left(X, Y_{1}, Y_{2}\right) \\
g\left(X, Y_{1}, Y_{2}\right) & \frac{r_{2} h}{X}
\end{array}\right| \\
& =\frac{r_{2} h}{k X}+g^{2}\left(X, Y_{1}, Y_{2}\right)>0, \\
M_{2} & =\left|\begin{array}{ccc}
\frac{1}{k} & -g\left(X, Y_{1}, Y_{2}\right) & 0 \\
g\left(X, Y_{1}, Y_{2}\right) & \frac{r_{2} h}{X} & \frac{r_{2} h}{2 X} \\
0 & \frac{r_{2} h}{2 X} & 0
\end{array}\right| \\
& =\frac{1}{k}\left(\frac{r_{2} h}{2 X}\right)^{2}>0,
\end{aligned}
$$

it is obvious that $d V / d t<0$. So the interior equilibrium point $E_{*}=\left(X_{*}, Y_{1 *}, Y_{2 *}\right)$ is globally asymptotically stable.

\section{Hopf Bifurcation}

Theorem 6. The dynamical system undergoes Hopf bifurcation around the interior equilibrium points $E_{*}$ whenever the critical parameter value $\beta=\beta_{H}$ is included in the domain:

$$
\begin{aligned}
D & =\left\{\beta_{H}\right. \\
& \in R^{+}: B_{1}(\beta) B_{2}(\beta)-\left.B_{3}(\beta)\right|_{\beta=\beta_{H}=0}, \text { with } B_{2} \\
& \left.>0,\left.\frac{B_{1}(\beta) B_{2}(\beta)-B_{3}(\beta)}{d \beta}\right|_{\beta=\beta_{H}} \neq 0\right\} .
\end{aligned}
$$

Proof. The characteristic equation of system (3) at $E_{*}=$ $\left(X_{*}, Y_{1 *}, Y_{2 *}\right) T$ is given by

$$
\lambda^{3}+B_{1} \lambda^{2}+B_{2} \lambda+B_{3}=0 .
$$

The conditions $B_{1}(\beta) B_{2}(\beta)-\left.B_{3}(\beta)\right|_{\beta=\beta_{H}}=0$ give

$$
b_{22}\left(b_{12} b_{21}+b_{23} b_{32}\right)-\left.b_{11}\left(b_{11} b_{22}-b_{12} b_{21}\right)\right|_{\beta=\beta_{H}}=0 \text {. }
$$

From (21) we should have

$$
\left(\lambda^{2}+B_{2}\right)\left(\lambda+B_{1}\right)=0
$$

which has three roots $\lambda_{1}=+i \sqrt{B_{2}}, \lambda_{2}=-i \sqrt{B_{2}}$, and $\lambda_{3}=$ $-B_{1}$.

Differentiating the characteristic (21) with regard to $\beta$, we have

$$
\begin{aligned}
\frac{d \lambda}{d \beta} & =-\left.\frac{\lambda^{2} \dot{B}_{1}+\lambda \dot{B}_{2}+\dot{B}_{3}}{3 \lambda^{2}+2 B_{1} \lambda+B_{3}}\right|_{\lambda=i \sqrt{B_{2}}} \\
& =\frac{\dot{B}_{3}-B_{2} \dot{B}_{1}+i \dot{B}_{2} \sqrt{B_{2}}}{2\left(B_{2}-i B_{1} \sqrt{B_{2}}\right)}=\frac{\dot{B}_{3}-\left(B_{2} \dot{B}_{1}+B_{1} \dot{B}_{2}\right)}{2\left(B_{1}^{2}+B_{2}\right)} \\
& +i \frac{\sqrt{B_{2}}\left(B_{1} \dot{B}_{3}+B_{2} \dot{B}_{2}-B_{1} \dot{B}_{1} B_{2}\right)}{2 B_{2}\left(B_{1}^{2}+B_{2}\right)} \\
& =-\frac{d\left(B_{1}(\beta) B_{2}(\beta)-B_{3}(\beta)\right) / d \beta}{2\left(B_{1}^{2}+B_{2}\right)}+i\left[\frac{\sqrt{B_{2}} \dot{B}_{2}}{2 B_{2}}\right. \\
& \left.-\frac{B_{1} \sqrt{B_{2}}\left(d\left(B_{1}(\beta) B_{2}(\beta)-B_{3}(\beta)\right) / d \beta\right)}{2 B_{2}\left(B_{1}^{2}+B_{2}\right)}\right] .
\end{aligned}
$$

Hence,

$$
\begin{aligned}
& \left.\frac{d}{d \lambda}(\operatorname{Re}(\lambda(\beta)))\right|_{\beta=\beta_{H}} \\
& \quad=-\left.\frac{d\left(B_{1}(\beta) B_{2}(\beta)-B_{3}(\beta)\right) / d \beta}{2\left(B_{1}^{2}+B_{2}\right)}\right|_{\beta=\beta_{H}} \neq 0
\end{aligned}
$$

and $B_{1}\left(\beta_{H}\right)<0$.

We can easily establish the condition of the theorem $(d / d \lambda)(\operatorname{Re}(\lambda(\beta))) \neq 0$, which completes the proof. 

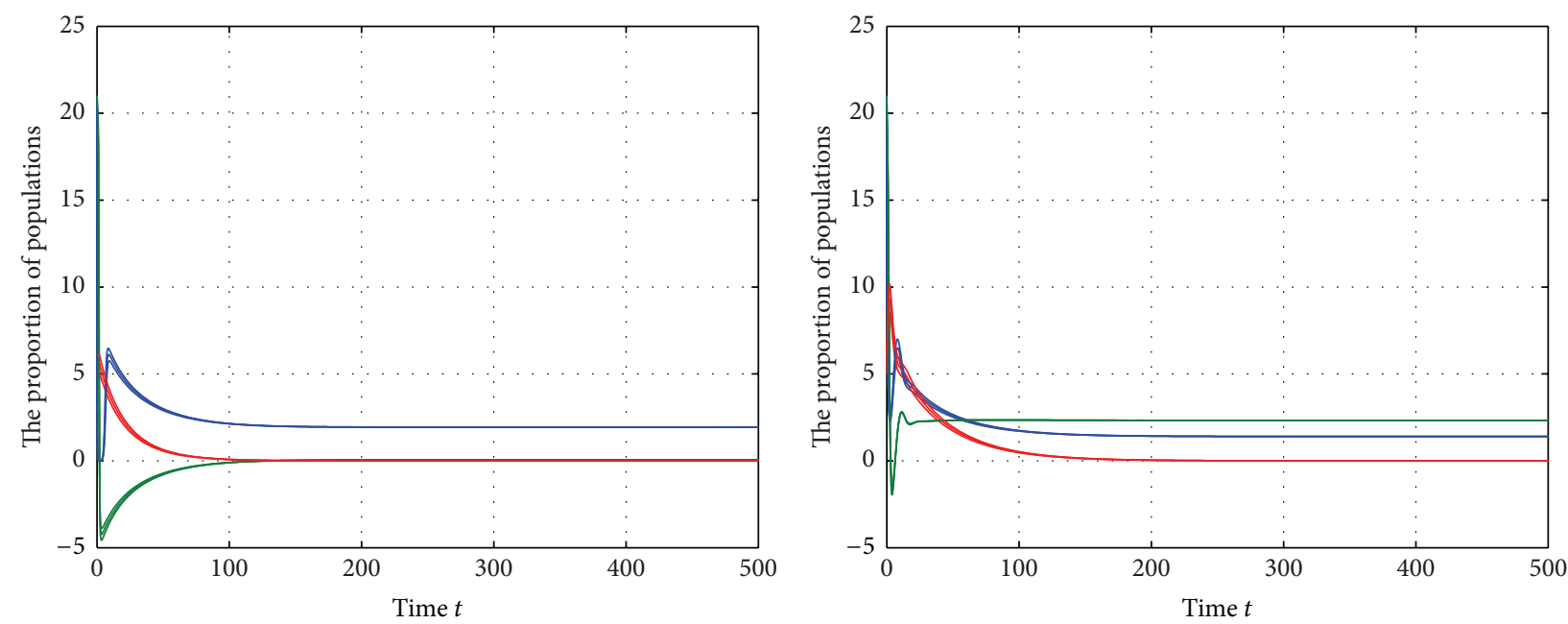

$X$
$-Y_{1}$
$-Y_{2}$

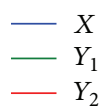

(a)

(b)

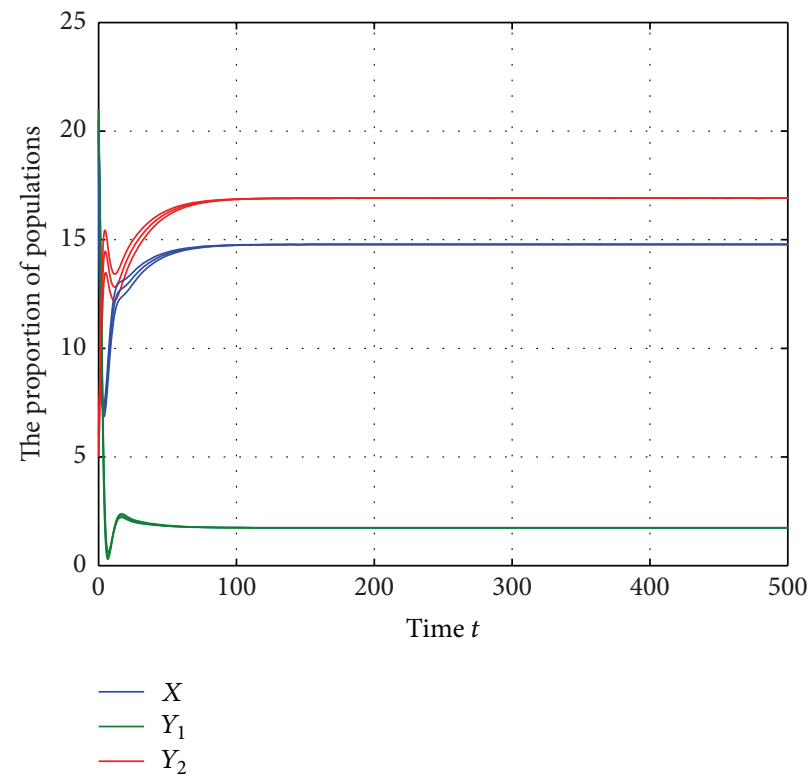

(c)

FIGURE 1: The local stability around all equilibriums of system (3).

\section{Number Simulations}

For the purpose of making qualitative analysis of the present study, numerical simulations have been carried out by making use of MATLAB.

The parametric values were given as follows: $r_{1}=0.5, k=$ 2, $a=0.3, r_{2}=0.002, h=5, \beta=0.003, d_{1}=0.03, d_{2}=0.04$, and $r_{2}-d_{1}=-0.028<0$. The eigenvalues of the Jacobian matrix at $E_{1}$ are $\lambda_{1}=-0.5, \lambda_{1}=-0.028$, and $\lambda_{3}=-0.04$, which satisfied the condition of the local asymptotic stability of axial equilibrium $E_{1}=(2,0,0)$ (see Figure 1(a)).

The parametric values were given as follows: $r_{1}=0.2$, $k=20, a=0.08, r_{2}=3, h=0.6, \beta=0.023, d_{1}=0.003$, $d_{2}=0.08$, and $k\left(r_{2}-d_{1}\right)\left(\beta r_{1}-a d_{2}\right)-r_{1} r_{2} h d_{2}=-0.1367<$ 0 , which satisfied the condition of the local asymptotic stability of planar equilibrium $E_{2}$. So the planar equilibrium $E_{2}=(1.3966,2.3254,0)$ is locally asymptotically stable (see Figure 1(b)).

The parametric values were given as follows: $r_{1}=0.2, k=$ $20, a=0.03, r_{2}=3, h=0.5, \beta=0.023, d_{1}=0.03, d_{2}=0.04$, and $\beta r_{1}-a d_{2}=0.0036>0, k\left(r_{2}-d_{1}\right)\left(\beta r_{1}-a d_{2}\right)-r_{2} h r_{1} d_{2}=$ $0.2018>0$, which satisfied the conditions for existence of interior equilibrium solution $E_{*} \cdot k r_{2} \beta r_{1}+k d_{1} a d_{2}+k a d_{2}^{2}-$ $k r_{2} a d_{2}-k d_{1} \beta r_{1}-2 r_{1} r_{2} h d_{2}-d_{2} k r_{1} \beta=-0.1626<0$; the conditions for the local asymptotic stability of interior equilibrium solution $E_{*}$ are well satisfied. Hence, the positive 


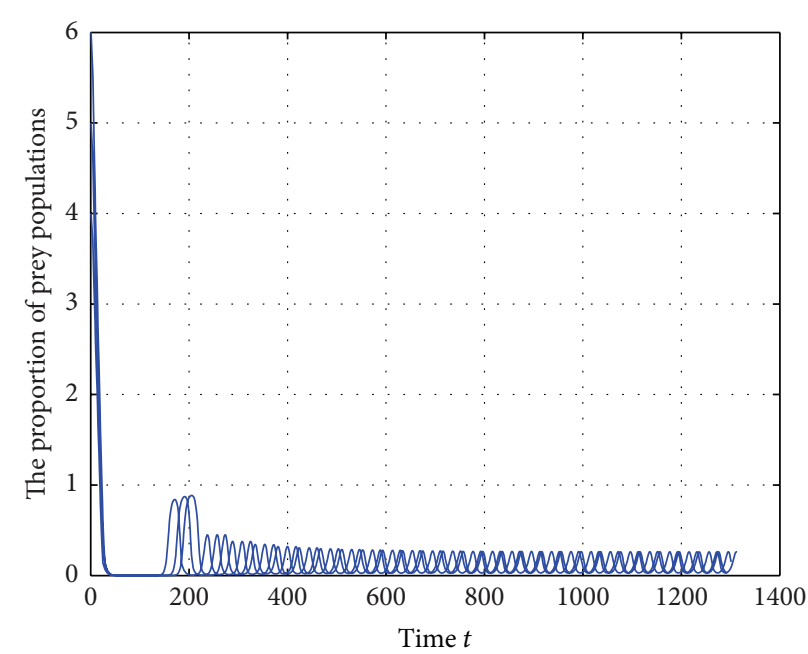

$-X$

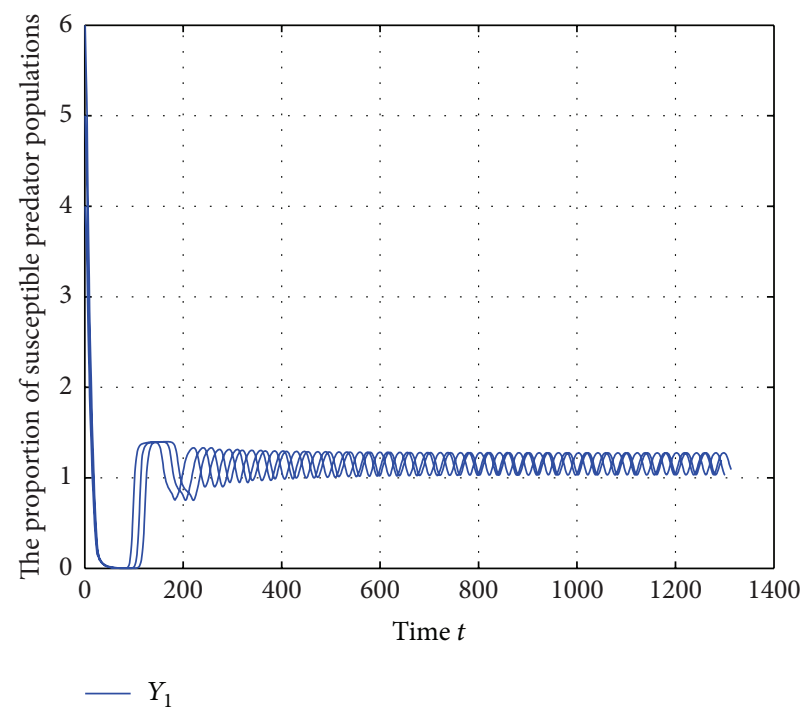

(b)

(a)

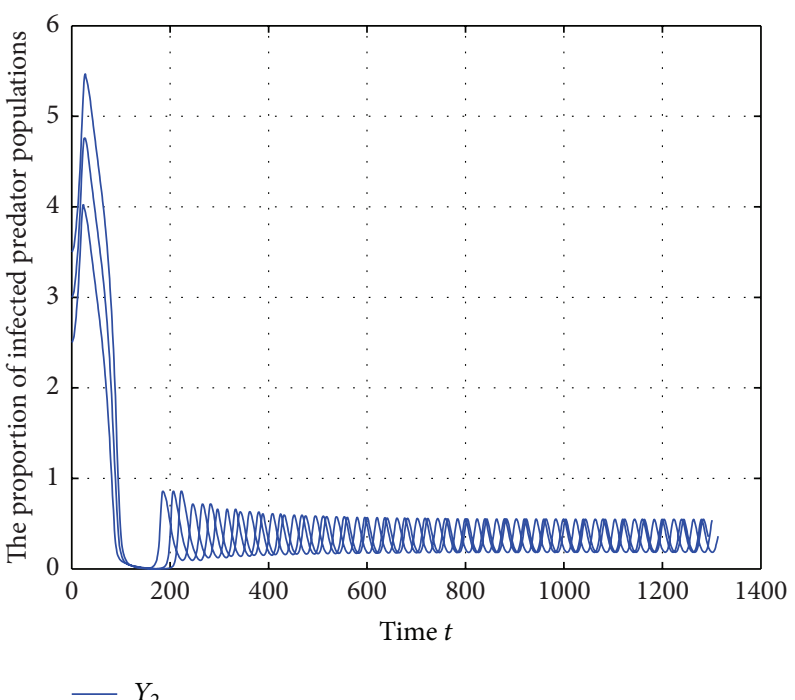

(c)

FIGURE 2: Hopf bifurcation around the interior equilibrium of system (3).

interior equilibrium $E_{*}$ is locally asymptotically stable in the neighborhood of $E_{*}$ (see Figure $1(c)$ ). Since the condition for the global asymptotic stability of $E_{*}$ holds good (see Theorem 5), the unique interior equilibrium solution $E_{*}=$ $(14.9542,1.7391,22.4433)$ is a global attractor (see Figure 3).

We have performed a bifurcation analysis of the model and obtained a critical value $\beta_{H}$. When the transmission coefficient from susceptible predator to infected predator $\beta$ passes through $\beta_{H}$, the system undergoes a Hopf bifurcation around the stationary state of coexistence (see Figure 2).

\section{Conclusions}

In this paper we have proposed and analyzed a Leslie-Gower ecoepidemiological model that divided the total population into three different populations, namely, prey $(X)$, susceptible predator $\left(Y_{1}\right)$, and infected predator $\left(Y_{2}\right)$. The conditions for existence and stability of the all equilibria of the system have been given. The bifurcation situations have also been observed around the interior equilibrium point.

The system has four equilibriums $E_{0}, E_{1}, E_{2}$, and $E_{*}$. We have obtained epidemiological threshold quantities for our model: $R_{0}^{1}=r_{2} / d_{1}, R_{0}^{2}=k\left(r_{2}-d_{1}\right)\left(\beta r_{1}-a d_{2}\right) / r_{2} h r_{1} d_{2}$, and $R_{0}^{3}=\left(k r_{2} \beta r_{1}+k d_{1} a d_{2}+k a d_{2}^{2}\right) /\left(k r_{2} a d_{2}+k d_{1} \beta r_{1}+\right.$ $\left.2 r_{1} r_{2} h d_{2}+d_{2} k r_{1} \beta\right)$. $E_{0}$ is unstable for all times. If $R_{0}^{1}<1$, the axial equilibrium $E_{1}$ is locally asymptotically stable. If $R_{0}^{2}<1$, the planar equilibrium $E_{2}$ is locally asymptotically stable. It is observed that the infected predator does not survive and could make the system free from disease. If $R_{0}^{2}>1$, the planer equilibrium $E_{2}$ is unstable, which is the conditions of 


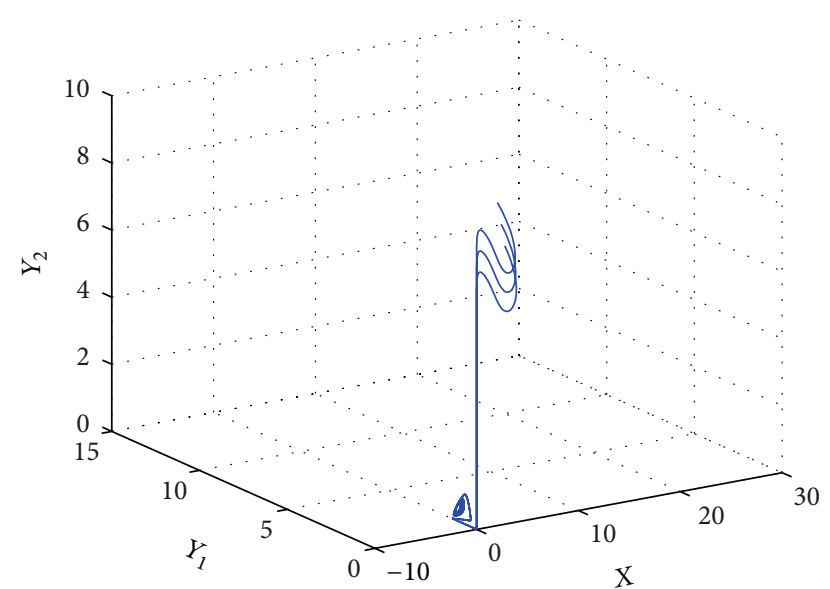

FIGURE 3: The global stability around the equilibriums $E_{*}$ of system (3).

the existence of the the interior equilibrium $E_{*}$. If $R_{0}^{3}<1$, the interior equilibrium $E_{*}$ is locally asymptotically stable, which means the global asymptotic stability.

We analyze the Hopf bifurcation around $E_{*}$, which means that the susceptible predator coexists with the prey and the infected predator showing oscillatory balance behavior.

\section{Conflict of Interests}

The authors declare that there is no conflict of interests regarding the publication of this paper.

\section{Acknowledgments}

The authors would like to thank the reviewers for their careful reading and constructive suggestions to the original paper that significantly contributed to improve the quality of the paper. And the authors gratefully acknowledge $\mathrm{Mr}$. Zhigang Chen for modifying the English grammatical errors in the revised paper. This work is supported by the National Sciences Foundation of China (10471040) and the National Sciences Foundation of Shanxi Province (2009011005-1).

\section{References}

[1] R. M. Anderson and R. M. May, "Regulation and stability of host-parasite population interactions: I. Regulatory processes," Journal of Animal Ecology, vol. 47, no. 1, pp. 219-247, 1978.

[2] E. Venturino, "Epidemics in predator-prey models: diseases in the prey," in Mathematical Population Dynamics: Analysis of Heterogeneity, vol. 1 of Theory of Epidemics, pp. 381-393, Wuerz Publishing, Winnipeg, Canada, 1995.

[3] M. Haque, J. Zhen, and E. Venturino, "Rich dynamics of LotkaVolterra type predatorprey model system with viral disease in Prey species," Mathematical Methods in the Applied Sciences, vol. 32, pp. 875-898, 2009.

[4] Y. Xiao and L. Chen, "Analysis of a three species ecoepidemiological model," Journal of Mathematical Analysis and Applications, vol. 258, no. 2, pp. 733-754, 2001.
[5] Y. Xiao and L. Chen, "Modelling and analysis of a predator-prey model with disease in the prey," Mathematical Biosciences, vol. 171, no. 1, pp. 59-82, 2001.

[6] J. J. Tewa, V. Y. Djeumen, and S. Bowong, "Predator-prey model with Holling response function of type II and SIS infectious disease," Applied Mathematical Modelling, vol. 37, no. 7, pp. 4825-4841, 2013.

[7] M. S. Rahman and S. Chakravarty, "A predator-prey model with disease in prey," Nonlinear Analysis: Modelling and Control, vol. 18, no. 2, pp. 191-209, 2013.

[8] M. Haque, S. Sarwardi, S. Preston, and E. Venturino, "Effect of delay in a Lotka-Volterra type predator-prey model with a transmission disease in the predator species," Mathematical Biosciences, vol. 234, no. 1, pp. 47-57, 2011.

[9] Y.-H. Hsieh and C.-K. Hsiao, "Predator-prey model with disease infection in both populations," Mathematical Medicine and Biology, vol. 25, no. 3, pp. 247-266, 2008.

[10] P. H. Leslie, "Some further notes on the use of matrices in population mathematics," Biometrika, vol. 35, pp. 213-245, 1948.

[11] A. Korobeinikov, "A lyapunov function for leslie-gower predator-prey models," Applied Mathematics Letters, vol. 14, no. 6, pp. 697-699, 2001.

[12] S. Sarwardi, M. Haque, and E. Venturino, "A Leslie-Gower Holling-type II ecoepidemic model," Journal of Applied Mathematics and Computing, vol. 35, no. 1-2, pp. 263-280, 2011.

[13] S. Sharma and G. P. Samanta, "A Leslie-Gower predator-prey model with disease in prey incorporating a prey refuge," Chaos, Solitons \& Fractals, vol. 70, pp. 69-84, 2015.

[14] M. A. Aziz-Alaoui, "Study of a Leslie-Gower-type tritrophic population model," Chaos, Solitons and Fractals, vol. 14, no. 8, pp. 1275-1293, 2002.

[15] F. Chen, L. Chen, and X. Xie, "On a Leslie-Gower predator-prey model incorporating a prey refuge," Nonlinear Analysis: Real World Applications, vol. 10, no. 5, pp. 2905-2908, 2009.

[16] W. Liu and C. Fu, "Hopf bifurcation of a modified leslie-Gower predator-prey system," Cognitive Computation, vol. 5, no. 1, pp. 40-47, 2013.

[17] J. K. Hale, Theory of Functional Differential Equations, Springer, New York, NY, USA, 1977. 


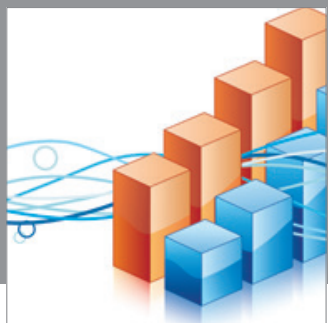

Advances in

Operations Research

mansans

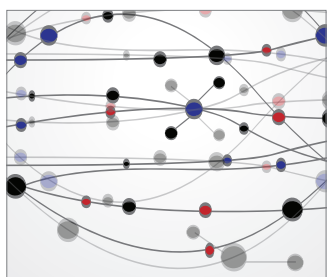

The Scientific World Journal
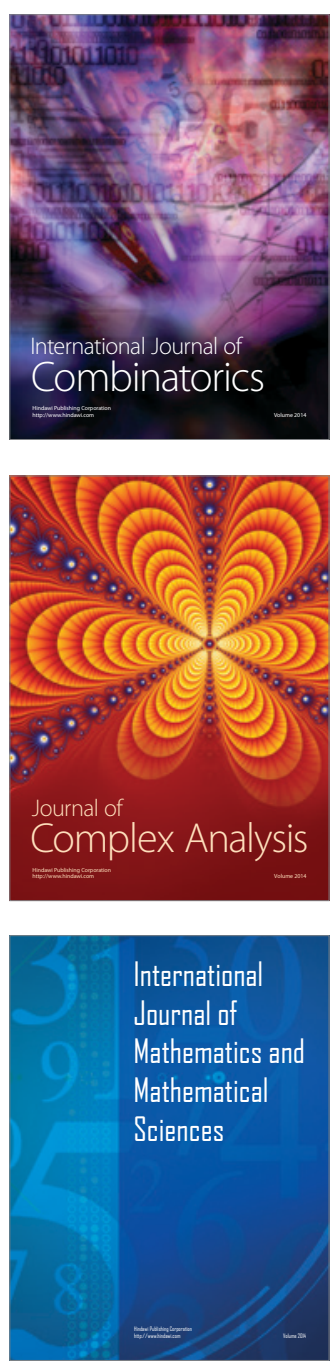
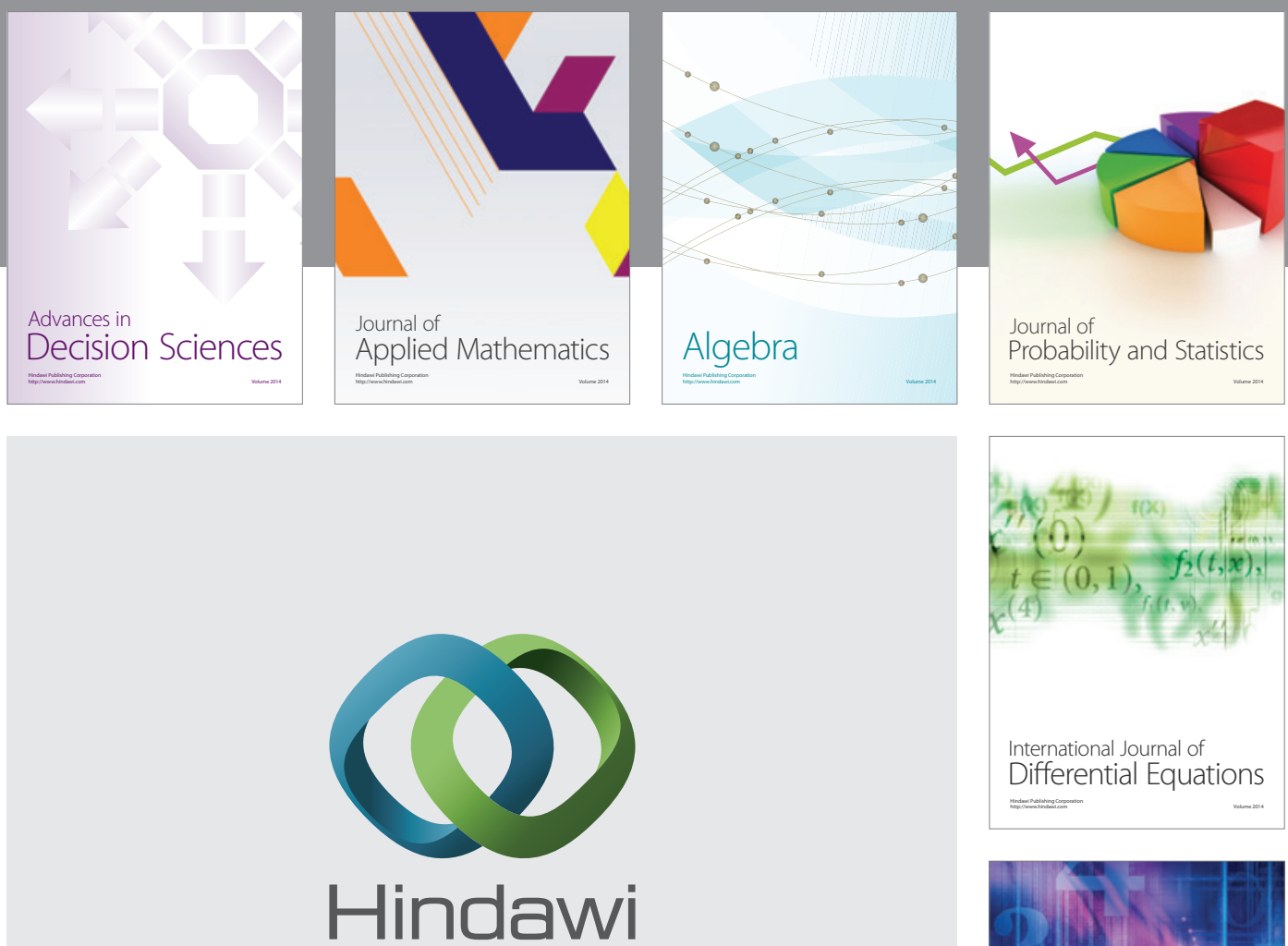

Submit your manuscripts at http://www.hindawi.com
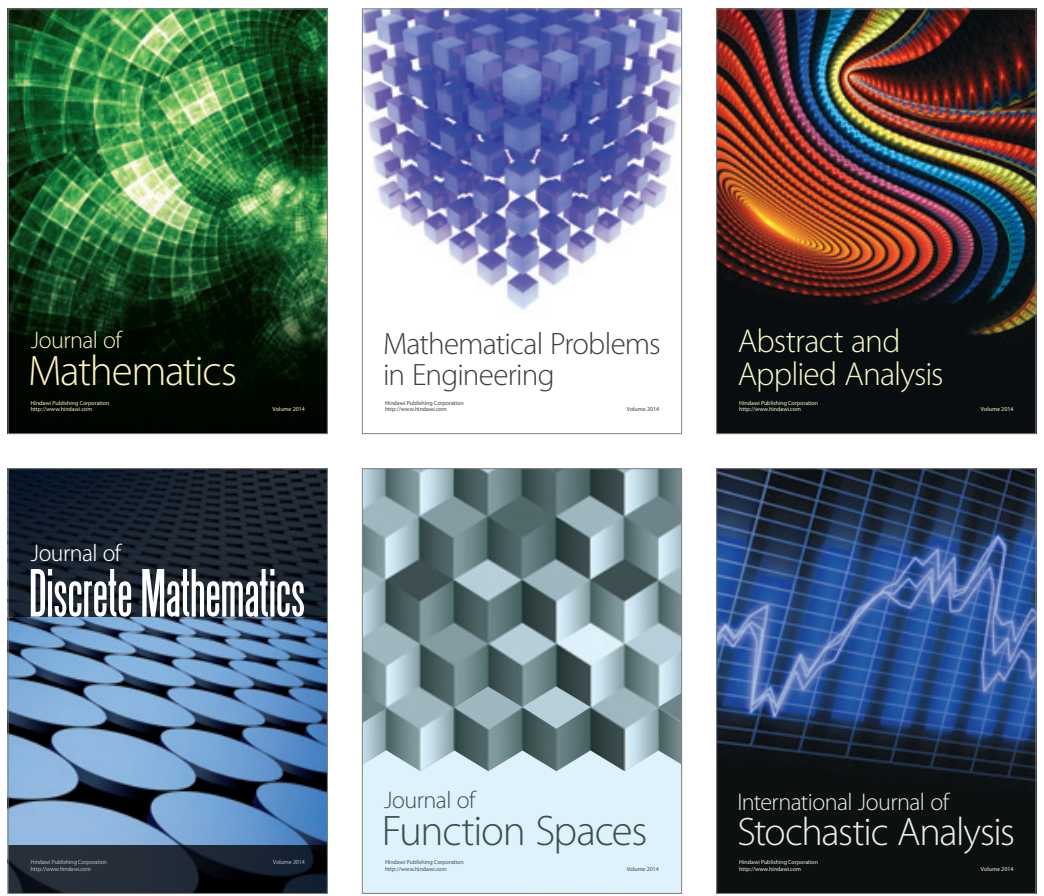

Journal of

Function Spaces

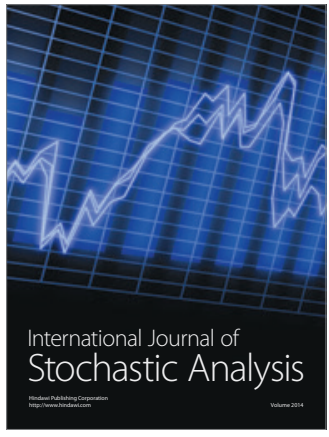

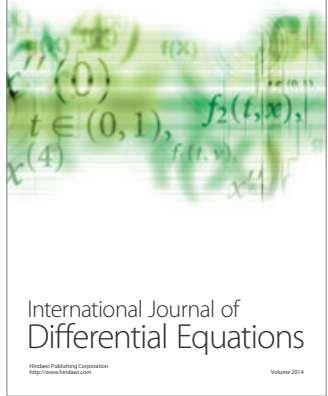
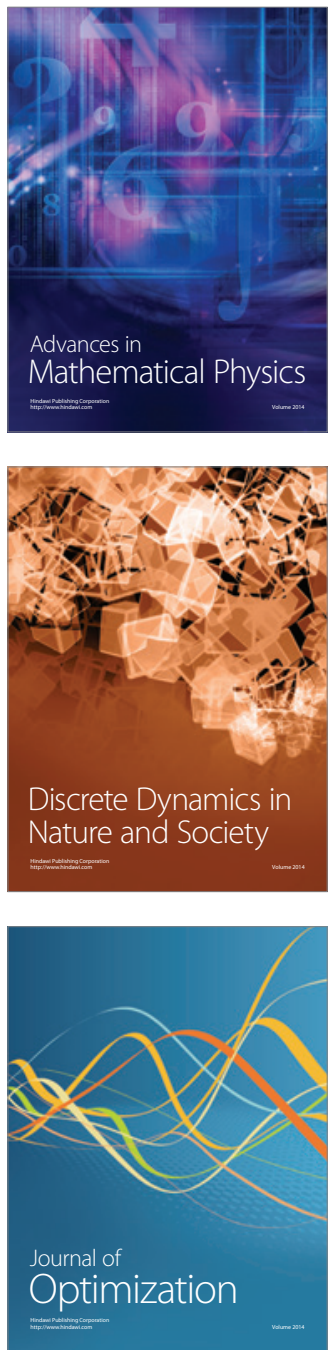